\title{
Zeitschriftenübersicht.
}

\section{Zeitschrift für die gesamte Neurologie und Psychiatrie.}

Red. von A. Alzheimer und M. Lewandowsky. Berlin, Jul. Springer. 1911.

Bd. 9. Heft 1. Henneberg, R., Pluriradikuläre Hinterstrangsdegeneration infolge von spinaler Cysticercusmeningitis. Krankengeschichte, Sektionsbefund und anatomische Schlussfolgerung. Schilder, P., Zur Frage herdgleichseitiger Hemiparesen bei Erkrankungen der hinteren SchädeIgrube. Hinweis auf Vorsicht bezüglich der Annahme cerebellarer Paresen. - Biondi, G., Histologische Betrachtungen an der Zirbeldrüse. - Enge, Zerstreuungen und Festlichkeiten in Irrenanstalten. Tanzfestlichkeiten und ähnliche Veranstaltungen sind möglichst zu vermeiden, dagegen soll mehr Sport im Freien getrieben werden. - Mollweide, K., Die Dementia praecox im Lichte der neneren Konstitutionspathologie. - Van der Torren, I., Psychosen und Psychoneurosen anf dem Boden einer iiberwertigen Idee. Besprechung zweier Krankheitsfälle. - Van der Torren, I., Der Entwurf der neuen Psychopathengesetze für die Niederlande. - Huber, O., Uber die degenerative Form der akuten Encephalitis und ihre Pathogenese. Ein Fall von akuter Encephalitis mit anatomischen und pathogenetischen Bemerkungen. - Stransky, E., Nachtrag zu dem Beitrag betreffend Dementia praecox.

Bd. 9. Heft 2. Schuppiu s, Über Schreibstörungen bei Epileptikern. Die Verwertbarkeit von Schreibversuchen bei Epileptikern im Anschluss an Krampfanfälle ist klinisch auf ein Minimum beschränkt. - Hauptmann, Die Levaditifärbung, eine spezifische Darstellungsmethode der senilen Pla. ques. - Friedman n, M., Zur Kenntnis der gehäuften nichtepileptischen Absencen im Kindesalter. 10 Fälle: kurze Dauer, geringe Intensität der Bewusstseinsstörung, Erschlaffung der Haltung, Aufwärtsdrehung der Augen, einige Schüttelbewegungen. Prognose: Heilung in den Pubertätsjahren. Schröder, G., Stoffwechselversuche bei Delixium tremens. In drei Fällen Verminderung der prozentualen Stickstoffmenge, in einem Falle unbedeutende Zunahme der im Ammoniak ausgeschiedenen Stickstoffmenge. Kafka, V., Über die Bedingungen nnd die praktische nnd theoretisehe Bedentung des Vorkommens Hammelblut lösender Normalambozeptoren und des Komplements im Liquor cerebrospinalis. Untersuchung uber die Beziehungen des Normalambozeptor- und Komplementgehalts an einem grösseren Material nebst theoretischen Erörterungen. - Haeffner, R., Beziehungen zwischen Menstruation und Nerven- and Geisteskrankheiten auf Grund der Literatur und klinischer Studien. I. Teil: Historischer Überblick. II. Teil: 
Untersuchungen an eigenem klinischen Material. Unter Anknüpfung an die Akromegalie kommt Verf. auf innere Sekretion, auf die Hypophyse usw. zu sprechen und schliesslich zu der Hypothese, es müsse ein nervöses Zentrum für die Menstruation bestehen.

Bd. 9. Heft 3. Marcu se, M., Ein Fall von vielfach homplizierter Sexualperrersion. Selbstbericht eines katholischen Geistlichen, veröffentlicht mit Einleitung, Nachtrag, Anmerkungen und Epikrise.-Heilbronner, K, Zwangsvorstellung und Psychose. Versuch, die Zwangsvorstellungen konventionell zu umgrenzen und Untersuchung über das genetische Verhältnis zwischen Zwangsvorstellungen und anderen gleichzeitig beobachteten Störungen, vor allem den depressiven Affekten. - Oberholzer, E., Spontaner Zahnausfall bei Paralyse. Klinische Beobachtung. - Lundborg, H., Der Erbgang der progressiven Myoklonusepilepsie (Myoklonie-Epilepsie s. Unverrichts familiäre Myoklonie). Die progressive Myoklonusepilepsie ist eine erbliche Krankheit, die mit grösster Wahrscheinlichkeit den Mendelschen Regeln folgt, rezessiv und monohybrid ist. Die Krankheit spaltet sich nicht in Myoklonie und Epilepsie, sondern wird als solche vererbt. Gurewitsch, M., Zur Differentialdiagnose des epileptischen Irreseins. Zugleich ein Beitrag zur Lehre von den kombinierten Psychosen. - Jas - pers, K.; Die phänomenologisehe Forsehungsrichtung in der Psychopathologie. Verfasser weist auf die Wichtigkeit phänomenologischer Einstellung des Psychiaters bei der Beurteilung Geisteskranker hin und fordert dazu auf, sich von jedem seelischen Phänomen, jedem Erlebnis. das in der Expluration der Kranken und ihren Selbstschilderungen zutage tritt, Rechenschaft zu geben. - Peiffer u. Albrecht, Zur Kenntnis der Harntoxizität des Mensehen bei rersehiedenen Krankheitsformen. Resultate der Untersuchungen an 13 Fällen von Epileptikern, die eine wesentlich gesteigerte Toxizität ihres Harns im Sinne der Temperaturreaktion erkennen lassen, ferner an einer Reihe verschiedener Erkrankungen.

Bd. 9. Heft 4. Lafora, Zur Frage der hereditären Paralyse des Erwachsenen (Dementia paralytica hereditaria tarda). Zngleich ein Beitrag zur histopathologischen Absonderung der jurenilen Paralyse. Die meisten Fälle von juveniler Paralyse zeigen neben den charakteristischen histopathologischen Veränderungen der Paralyse andere Zeichen, welche auf eine Entwicklungshemmung des Nervengewebes hindeuten. Es gibt Fälle von hereditärer Paralyse im Erwachsenenalter. In Fällen von Paralyse der Erwachsenen, in denen die histo-pathologische Untersuchung Entwicklungsstörungen andeutende Veränderungen feststellt, sind wir berechtigt, die Diagnose einer Dementia paralytica hered. tard. zu stellen. Fälle juveniler Paralyse ohne Entwicklungsstörungen des Nervengewebes, können wir als placentare Virusübertragung zur Zeit fortgeschrittener Gewebselemententwicklung ansehen. - Mees, R. A., Ein röhrenförmiges Gliom des Rückenmarks mit regionären Metastasen. Klinischer Fall mit Autopsiebericht und histopathologischer Untersuchung. - Szécsi, St., Weitere Beiträge zur Cytologie des Liquor cerebrospinalis. Über die sogenannte Degeneration der Zellen. Degeneration der Liquorzellen ist nur ein Kunstprodukt, entstanden durch Färbe- oder Härtungsmethoden. Labilität und Farbgierigkeit der Liquorzellen ohne pathologische Bedeutung. Kleinheit 
der Lymphoidzellen, wohl auf ein unbekanntes schädigendes Agens zurückzuführen. - Sträussler, E., Abszess im rechten Schläfenlappen bei einem Linkshänder mit sensorischer Aphasie. Klinischer Fall mit Autopsie. Beim Linkshänder ist der Sitz des Sprachzentrums in der rechten Hemisphäre zu suchen. Die Lokalisation verbleibt auch, wenn das betreffende Individuum mit der rechten Hand schreiben odes alltägliche Hantierungen mit der rechten Hand vorzunehmen lernt. Hinweis auf die Fruchtlosigkeit der Zweihandkultur. - Sträussler, E., Ein Fall von passagerer. systematischer Sprachstörung bei einem Polyglotten, verbunden mit rechtsseitigen transitorischen Gehörshalluzinationen. - Fischer, O., Ein Beitrag zur Frage des cerebralen Fiebers. Klinisch beobachteter Fall, therapeutisch mit geringer Hyoscin-Morphiummenge beeinflusst. - Cerletti, Über verschiedene Encephalitis- und Myelitisformen bei an Staupe erkrankten Hunden. Zur Kenntuis der sog. progressiven Paralyse der Hunde. Historisches Untersuchungsmaterial. Katarrhalische Form der Staupe, nervöse Staupe, Hundeparalyse, epileptische Hunde, Zusammenfassung. - Schellmann, Über Trinkerfürsorgestellen. Zu dem Aufsatz von Dr. R. Hirschfeld dieser Zeitschrift, Heft 8. S. 659. 1912.

Bd. 9. Heft 5. Krabbe, K., Über Paralysis agitans-ähnlichen. Tremor bei Dementia paralytica. Klinische Beobachtung. — Luther, A., Über das Vorkomuton chronischer Alkoholhalluzinosen. Es gibt eine chronische Alkoholhalluzinose, selten in komplizierter Form. Häufiger sind Fälle von Alkoholismus kombiniert mit Dementia praecox. Auftreten vielleicht regionär verschieden. - Heilig a. Steiner, Zur Kenntnis der Entstehungsbedingungen der genuinen Epilepsie. Untersuchungen an 567 Soldaten. In Linkshänderfamilien ist die genuine Epilepsie desgleichen der Sprachstörung viel häufiger als bei Rechtshändern. Dies Zusammentreffen ist wohl eine durch Schädigung der linken Hemisphäre bedingt.

Bd. 10. Heft 1 u. 2. Schilder, P., Zur Kenntnis der sogenannten diffnsen Sklerose (über Encephalitis periaxialis diffusa). Encephalitis periax. diff. bezeichnet Verf. eine diffuse Erkrankung des Hemisphärenmarks im Kindesalter, die unter verschiedenen Krankheitsbildern verläuft, tötlich ist, pathogenetisch, besonders in der akuten Form der multiplen Sklerose nahesteht und zur Zeit noch unbekannter Ätiologie ist. Pfeifer, B., Weitere Erfahrungen über Hirnpunktion bei Hirntumoren und Epilepsie. An Hand einer grösseren Anzahl von Fällen, die nach Hirnpunktion teils operiert, teils nicht operiert wurden, sowie der entsprechenden Sektionsbefunde, weist Verf. auf die diagnostisch günstigen Resultate der Hirnpunktion hin. Zum Schluss bespricht er noch Indikationen und Kontraindikationen sowie therapeutische Erfolge bei Epileptikern: - Heilig, Über Alkoholpsychosen. Eingehende Arbeit nach statistischen, ätiologischen, pathogenetischen und klinisehen Gesichtspunkten geordnet (Material der psychiatrischen und Nervenklinik Strassburg). Is serlin, M. und Lotmar, F., Über den Ablauf einfacher, willkürlicher Bewegungen bei einigen Nerven- und Geisteskranken. - Schaffer, K., Hirnpathologische Beiträge. Kasuistische sowie pathologisch-auatomische Arbeit über Pseudobulbärparalyse und Hemiplegie.

Bd. 10. Heft 3. Wyss, W. v., Verbrechen vor oder im Beginn 
der Dementia praecox als Inhalt der Psychose. - Haugh, W. H., Die Wirkung von Alkoholgaben auf die Wassermannsche Reaktion. Experimentelle Arbeit - Sittig, 0 ., Über eine besondere Form akuter, herdförmiger Destruktion des nervösen Gew ches im Gehirn bei Tuberkulose (akute, nicht eiterige Encephalitis). Es müssen entzündliche und degenerative Prozesse in der Anatomie der Encephalitis unterschieden werden. Hallager, Über die postparoxysmale Albuminurie und Polyurie der Epileptiker. Bemerkungen zu einer früheren Arbeit des Verf. - Schob, F., Zur pathologischen Anatomie der juvenilen Form der amaurotischen Idiotie. Krankengeschichte mit Sektionsbefnnd und vorzliglichen mikroskopischen Bildern. - Skliar, N., Zum Wesen der Hysterie.

Bd. 10. Heft 4 u. 5. Na ecke, P., Einteilnng der (habntuell) Antisozialen und der mehr oder minder moralisch Defekten. - Schultze, E., Krankhafter Wandertrieb, räumlich beschränkte Taubheit für bestimmte Töne und tertiäre Empfindungen beim Psychopathen. - Schuppius, Beitrag zur Vagabundenfrage. Kausale Beziehungen zwischen Psychose und unsozialer Lebensfïhrung, an Hand von Krankengeschichten erörtert, Notwendigkeit des Verbleibens von psychopathischen Landstreichern in Arbeitsresp. Armenhäusern, Forderung einer psychiatrischen Vorbildung der betreffenden Anstaltsärzte, Erhebung genauer Anamnese jedes Landstreichers, eventuelle Unterbringung in Irrenanstalten. - Rohde, M., Zur Genese ron ,Antällen“6 und diesen nabestehenden Zuständen bei sogen. Nervösen. Wichtigkeit des vasomotorischen Momentes bei verschiedenen nervösen Erscheinungen. - Ebstein, E., Über die Perkussion des Schädels. Verfasser fügt seinen früheren historisch-klinischen Abhandlungen eine neue hinzu, die dank seiner eingehenden Literaturkenntnis interessant ist. Er weist darauf hin, dass trotz der derzeitigen Unvollkommenheit der Schädelperkussion dieser nicht der letzte Platz in der Reihe der Untersuchungsmethoden gebührt. - Strasmann, R., Myoklonie bei Tabes. Verf. liefert einen Beitrag zur Frage der Myoklonie und beschreibt ein sehr gut beobachtetes Krankheitsbild eines Falles von Tabes mit myoklonischen Zuckungen. - Strohmeyer, W., Kinderhysterie imit schweren Störungen der Lage- und Bewegungsempfindungen. Interessantes polysymptomatisches Krankheitsbild. - Boas, H. und Neve, G., Untersuchnngen ïber die Weil-Kafkasche Hämolysinreaktion in der Spinalfiüssigkeit. Experimentelle Arbeit.

W. Röpert-Leipzig.

\section{Monatsschrift für Psychiatrie und Neurologie.}

Herausgegeben von Prof. Dr. Th. Ziehen. Berlin.

Bd. 31. Heft 2-6. H. Fabritius (Helsingsfors), Zur Frage nach der sensiblen Leitung im menschlichen Rückenmark. Zum kurzen Referat uicht geeignet.

Bd. 31. Heft 3-5. Pfersdorff (Strassburg), Zur Gruppierung der sprachlichen Assoziation.

Bd. 31. Heft 5 (Mai 1912). Hans Bergংr (Jena), Experimentelle Untersnchungen über die Einwirknng ron Gehirnblutungen. Gehirnembo- 
lien, epileptischen Anfällen und Gehirnerschütterungen anf die Blutzirkulation im Gehirn. An der Hand von an Hunden gewonnenen Kurven wird u. a. gezeigt, dass bei den genannten vier Zuständen sicherlich keine allgemeine Kontraktıon der Rindengefässe statt hat, sondern dass vielmehr von einer allgemeinen Gefässerweiterung gesprochen werden muss.

Bd. 31. Heft 5 u. 6. K. Krause (Berlin), Zur Histopathologie der Gehirncysticerkose. Von Resten abgestorbener Cysticerken gehen beständig Reizerscheinungen aus, die einen chronischen Entzündungsprozess unterhalten, dessen diffuse Verbreitung sich in den weichen Häuten des Hemisphärén, des Hirnstamms, der Medulla oblongata, ja im Rückenmark nachweisen liess. - Zum Schluss: Schilderung des klinischen Symptomenkomplexes.

\section{Monatsschrift für Psychiatrie und Neurologie.}

Herausgegeben von Prof. Dr. K. Bonhoeffer.

Bd. 32. Heft 1 (Juli 1912). W. Tintemann (Göttingen): Stoffwechseluntersuchungen an Kranken mit epileptischen und epileptiformen Krampfanfällen. Auch bei der nicht genuinen Epilepsie lassen sich Störungen im intermediären. Stoffwechsel nachweisen. - A. Romagna-Manoia, Über die Neurorezidive der lnetischen Affektionen des Nervensystems. Zu kurzem Referate nicht geeignet. - A. Gregor (Leipzig), Űber Nebenwirkungen von Schlafmitteln: an der Hand von Blutdruckversuchen (Us koff scher Apparat) und Beobachtung der Pulsfrequenz mit Veronal, Proponal, Neuronal, Medinal Isopral usw.). - Otto Klieneberger (BreslauKönigsberg), Zur Erweiterung der Wassermunnschen Methode, Liquorund Komplementauswertungsverfahren. Im wesentlichen Bestätigung des Hauptmannschen Auswertungsverfahren. - N. M. Rudnitzky (Charkow), Neurasthenie und Tuberkulose. Ein Versuch aus dem modernen Begriff der Neurasthenie eine Krankheitsform organischen Ursprungs auszuscheiden. Berücksichtigt besonders die bisher dem Bilde der Neurasthenie versteckten Lungenspitzenkatarrhe, Pleuritiden usw.

$$
\text { Erich Ebstein (Leipzig). }
$$

